\title{
A scalable galvanic approach to microswimmer synthesis
}

\author{
Maximilian R. Bailey, ${ }^{* a}$ Nico Reichholf, ${ }^{a}$ Anne Flechsig, ${ }^{a}$, Fabio Grillo, ${ }^{a}$ and L. Isa ${ }^{* a}$ \\ ${ }^{a}$ Laboratory for Soft Materials and Interfaces, Department of Materials, ETH Zürich, \\ Vladimir-Prelog-Weg 5, 8093 Zürich, Switzerland \\ *To whom correspondence should be addressed: \\ E-mail: maximilian.bailey@ mat.ethz.ch; \\ E-mail: lucio.isa@mat.ethz.ch
}

\begin{abstract}
Microswimmers are small particles capable of converting available energy sources into propulsion owing to their compositional asymmetry and are promising for applications ranging from targeted delivery to enhanced mixing at the microscale. However, current fabrication techniques demonstrate limited scalability and/or rely on the excessive use of expensive precursor materials. Here, a scalable Pickering-wax emulsion technique is combined with galvanic electrochemistry, to grow platinum films from copper nanoparticles asymmetrically seeded onto $\mathrm{SiO}_{2}$ microparticle supports. In this manner, large quantities of $\mathrm{Pt}_{-} \mathrm{SiO}_{2}$ Janus microswimmers are obtained. Utilising copper as a templating material not only reduces synthesis time, material costs, and toxic waste, but also facilitates the further extension of this methodology to a range of functional materials. This electrochemical approach builds upon previous attempts to overcome the current limitations in microswimmer synthesis and offers exciting opportunities for their future development.
\end{abstract}


Artificial microswimmers are small synthetic particles that transform spatially uniform energy sources, e.g. external fields or chemical species, into propulsive motion (1). As they continuously consume energy, they are intrinsically "out-of-equilibrium" materials, displaying a range of collective phenomena not observed in systems at thermodynamic equilibrium (2). Beyond their fundamental appeal, microswimmers are interesting for a range of applications ranging from targeted drug delivery (3), to environmental remediation (4), and even energy conversion (5), as they enable directed transport of matter at small scales (6) and induce the mixing and pumping of fluids without external agitation (7). Janus microswimmers are arguably the simplest class of microswimmers. In Janus microswimmers, surface patches with different physicochemical properties induce the self-generation of asymmetric gradients, in turn imparting propulsion (8). Among those, chemical microswimmers exploit the catalytic decomposition of chemical fuel, e.g. hydrogen peroxide catalysed by a platinum surface, to generate local chemical gradients. However, even these simple microswimmers suffer from a very low throughput, as attaining patchy surfaces for large quantities of catalytically-active particles is synthetically challenging (9) and physical fabrication methods, such as metal deposition, are only able to provide minute particle quantities at the expenses of a large waste of catalytic material (10). In a recent advance in the state-of-the-art of microswimmer synthesis, Archer et al. demonstrated the scalable fabrication of Janus chemical microswimmers by functionalising particles confined on the surface Pickering-wax emulsion droplets with a two-step nanoparticle seeding and film-growth protocol (11). However, this approach involves a poorly controlled reduction of platinum $(\mathrm{Pt})$ nanoparticles from solution, requiring a significant use of expensive and toxic platinum salt precursor, as well producing undesirable nanoparticle waste (12). Furthermore, the approach as described is limited to the growth of platinum films.

Here, we combine the Pickering wax-emulsion technique with galvanic electrochemistry to obtain Pt metal films from copper nanoparticle seeds asymmetrically grown on $\mathrm{SiO}_{2}$ micropar- 

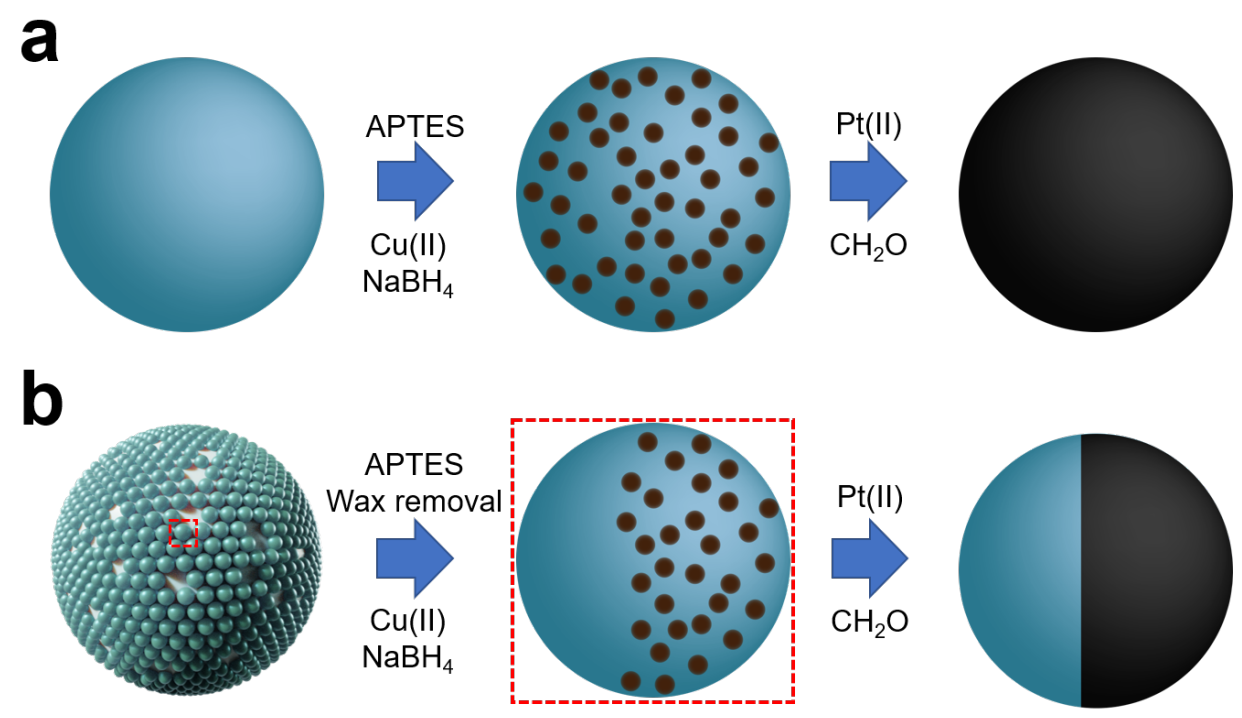

Figure 1: Schematic of the synthetic approach used to grow uniform and asymmetric Pt films: a. Bulk modification of the $\mathrm{SiO}_{2}$ microparticles with uniform Pt films b. Asymmetric growth of $\mathrm{Pt}$ films on $\mathrm{SiO}_{2}$ microparticles via $\mathrm{SiO}_{2}$-wax colloidosomes obtained with the Pickering emulsion technique

ticle supports (Figure 1). In particular, we exploit the rapid complexation of $\mathrm{Cu}(\mathrm{II})$ by amines present on the surface of the $\mathrm{SiO}_{2}$ microparticle supports. Copper nanoparticles are thus grown directly on the $\mathrm{SiO}_{2}$ upon addition of a strong reducing agent, providing greater control over nanoparticle growth and minimising the formation of excess nanoparticles. Using copper as a sacrificial template not only reduces nanoparticle waste and the use of expensive and toxic metal precursors, but copper can also be galvanically replaced with a range of metals, promising an economical and versatile approach to fabricate microrobots with different functionalities (13). By tracking the motion of the Pt microswimmers, we finally confirm that a clear asymmetry in the Pt films, obtained here using Pickering-wax emulsions, is a necessary precondition for appreciable microswimmer motion.

We first develop a protocol for growing platinum films on $\mathrm{SiO}_{2}$ microparticles without asymmetry, i.e. under bulk mixing conditions. Our method relies on the rapid complexation of cop- 
Bulk Functionalisation
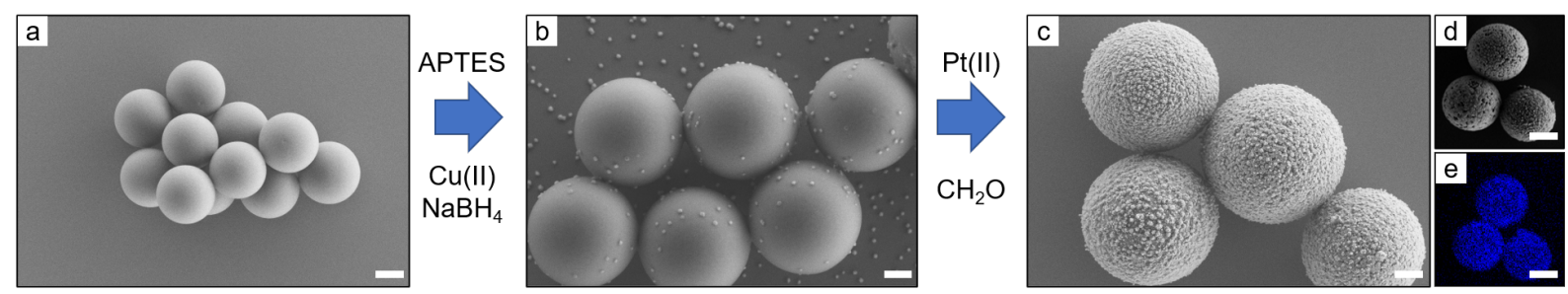

Asymmetric Functionalisation
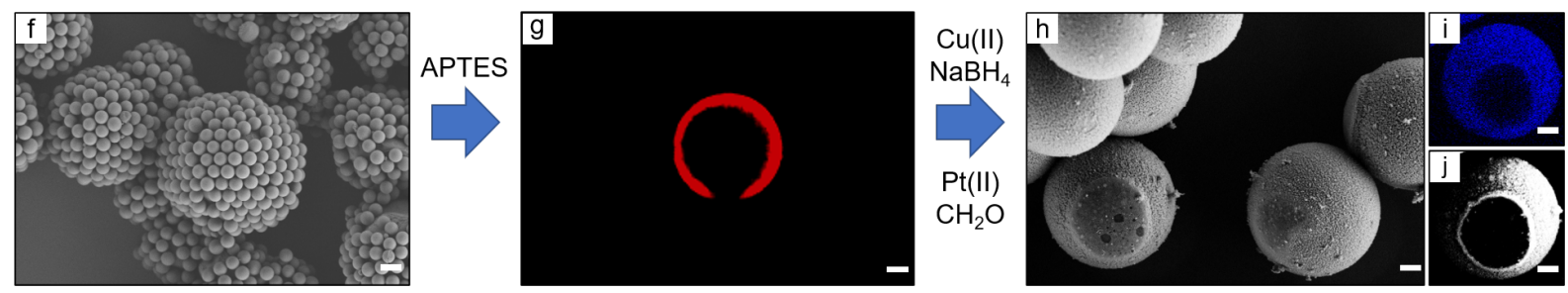

Figure 2: Growth of homogeneous and asymmetric Pt films: a. $\mathrm{SiO}_{2} 4.16 \mu$ m particles (scale bar $2 \mu \mathrm{m})$ b. $\mathrm{SiO}_{2}$ particles after copper complexation and reduction. Note that many seeds detach during the drying process for SEM sample preparation (scale bar $1 \mu \mathrm{m}$ ) c. $\mathrm{Pt}_{-} \mathrm{SiO}_{2}$ bulkmodified particles (scale bar $1 \mu \mathrm{m}$ ) d. SEM image and e. SEM-EDX indicating presence of a uniform Pt film (scale bar $2.5 \mu \mathrm{m}$ ). f. $\mathrm{SiO}_{2}$-wax colloidosomes prepared with $4.16 \mu \mathrm{m}$ particles (scale bar $5 \mu \mathrm{m}$ ) g. Janus APTES-modified $\mathrm{SiO}_{2}$ particle, further modified with NHS-ester dye after removal of the wax. The dye selectively binds to the APTES groups on the particle (scale bar $0.75 \mu \mathrm{m}$ ) h. Patchy $\mathrm{Pt}_{-} \mathrm{SiO}_{2}$ particles (scale bar $0.5 \mu \mathrm{m}$ ) i. SEM image and j. SEM-EDX indicating the presence of an asymmetric Pt film (scale bar $0.75 \mu \mathrm{m}$ ).

per ions by amine groups added to the $\mathrm{SiO}_{2}$ microparticle surface (14), which reduces the time required for synthesis. By first complexing the ions onto the microparticle supports before performing the reduction step, the uncontrolled formation of metal nanoparticles upon addition of a strong reductant is virtually eliminated. An aqueous $\mathrm{CuSO}_{2}$ solution is added drop-wise to an APTES-modified $\mathrm{SiO}_{2}$ microparticle suspension under magnetic stirring. The solution is then washed by centrifugation to remove free ions from solution. The particles retain a light blue colour from the complexed copper ions. An aqueous $\mathrm{NaBH}_{4}$ solution is then added dropwise to this mixture, which is left to react under magnetic stirring in an $\mathrm{N}_{2}$ environment. The solution rapidly turns a dark-brown colour upon addition of the $\mathrm{NaBH}_{4}$, indicating the formation 
of $\mathrm{Cu}^{0}$ nanoparticles, which are also visualised by SEM (Figure 2b). The resulting $\mathrm{Cu}-\mathrm{SiO}_{2}$ particles are then washed in degassed double-distilled water, retaining a brown colour from the $\mathrm{Cu}^{0}$ nanoparticles attached onto their surface.

The copper nanoparticles seeded onto the $\mathrm{SiO}_{2}$ microparticle supports act as a template for the electroless deposition of a platinum film (13). $\mathrm{H}_{2} \mathrm{PtCl}_{6}$ is added to the $\mathrm{Cu}-\mathrm{SiO}_{2}$ particle suspension and mixed in an N2 atmosphere. From the respective half-cell potentials of platinum and copper, a spontaneous galvanic replacement reaction between $\mathrm{Cu}^{0}$ and $\mathrm{Pt}$ is predicted. After 30 minutes, formaldehyde is added as a weak reducing agent to assist the autocatalytic film growth of Pt from the Pt-Cu seeds on the $\mathrm{SiO}_{2}$ microparticle support (11). The (yellow) mixture is left to react overnight in an $\mathrm{N}_{2}$ atmosphere under magnetic stirring. The (now clear) suspension is then centrifuged with double-distilled water, and the resulting particles are black, indicating a successful exchange with Pt and subsequent film growth (Figures 2c-e). We note that by increasing the Pt concentrations and reaction times used in the film growth step, the functionalised particles possess thicker and more uniform Pt films. FIB-SEM images show the likely mechanism of film growth, as Pt islands gradually fuse before increasing in thickness (Figure 3). We finally mention here the importance of following the colour changes of the particles and solutions as an indicator for the success of the various reaction steps.

After establishing a protocol for the growth of uniform Pt films on $\mathrm{SiO}_{2}$ microparticles, we extend the method by introducing asymmetry to the APTES surface modification of the $\mathrm{SiO}_{2}$ microparticles via Pickering-wax emulsions, in order to obtain chemical microswimmers (11). The Pickering-wax emulsion approach used here consists in decorating the surface of molten wax droplets in a hot aqueous medium with $\mathrm{SiO}_{2}$ microparticles. The particles are irreversibly adsorbed at the water-wax interface in the presence of a surfactant, and are immobilised when the wax is solidified. The particle surface immersed in the wax is then protected from chemical modifications that are subsequently carried out in the aqueous phase. We prepare the Picker- 


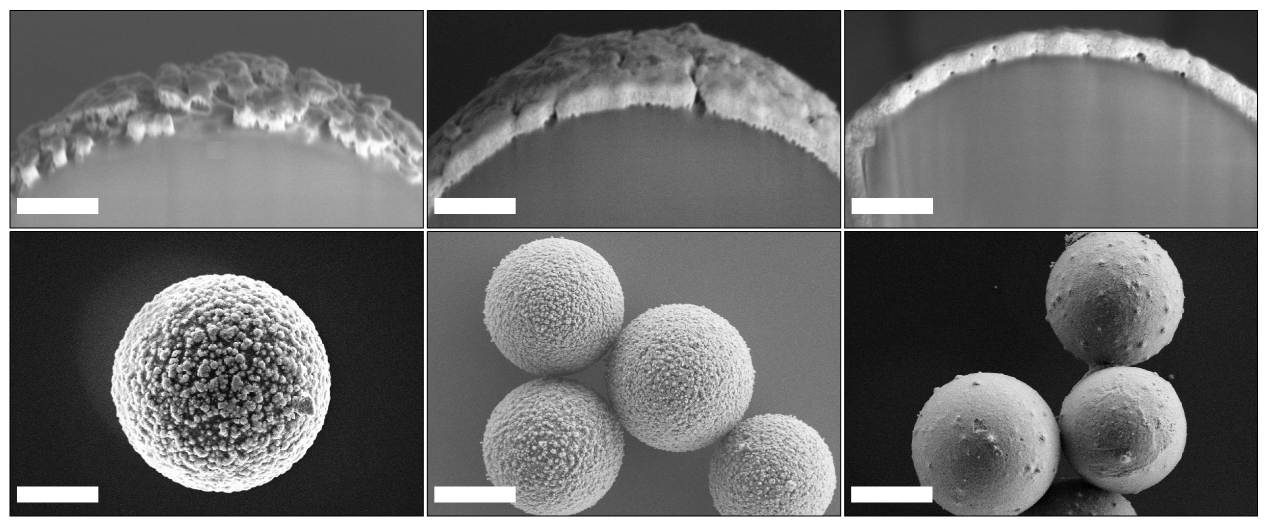

Figure 3: HR-SEM and FIB-SEM images of various film morphologies and thicknesses observed. Top row: FIB-SEM images of the particles with morphologies similar to those of the bottom row. Film thicknesses (left to right): $130 \mathrm{~nm}, 160 \mathrm{~nm}, 210 \mathrm{~nm}$. Scale bars of the top and bottom rows are $0.5 \mu \mathrm{m}$ and $2.0 \mu \mathrm{m}$ respectively

ing emulsions by adapting the methodology described by Perro et al (15). $\mathrm{SiO}_{2}$ particles are dispersed in aqueous didodecyldimethylammonium bromide (DDAB) solutions with concentrations selected to provide monolayer coverage of the surfactant on all particles (16). Solid wax is added to the suspension heated at $75^{\circ} \mathrm{C}$, and then subjected to a two-step vigorous homogenisation protocol (17). The hot wax-water Pickering emulsion is then rapidly cooled in an ice-bath to obtain solidified $\mathrm{SiO}_{2}$-wax colloidosomes (Figure 2f). The colloidosome solution is then cleaned by sedimentation with distilled water, a $0.1 \mathrm{M} \mathrm{NaCl}$ solution to remove the DDAB cationic surfactant, then water once more to remove the salt (18). The flexibility of the approach is demonstrated by producing emulsions with various particle sizes (Figure S2, Table S1), suggesting an adaptable method to obtain microswimmers with different physical properties.

The cleaned colloidosomes are then dispersed in an aqueous $\mathrm{NH}_{4} \mathrm{OH}$ solution, to which APTES $(360 \mathrm{uL})$ is added dropwise. The solution is then gently agitated using an orbital mixer. APTES modification is conventionally carried out in alcohols for greater control over the silanisation process, however, we find that the produced colloidosomes degrade when placed in or- 
ganic solvents (see Text S2, Figure S4). We therefore carry out the asymmetric APTES modification of the $\mathrm{SiO}_{2}$ microparticles in water (15), and use a large excess of APTES to account for losses from self-condensation reactions. We note that the primary objective here is the presence of amine groups for copper complexation on the $\mathrm{SiO}_{2}$ surface, and thus a smooth monolayer coverage of APTES molecules is of lesser import. To remove excess APTES, weakly attached particles, and any potential side-products from the APTES modification process, the APTES modified colloidosomes are then filtered and washed extensively with twice-distilled water. The colloidosomes are then dispersed in chloroform before filtration to remove the protecting wax masks (Figure 2g). Finally, the asymmetrically APTES-modified $\mathrm{SiO}_{2}$ particles are plated with $\mathrm{Pt}$ as described previously to obtain $\mathrm{Pt}-\mathrm{SiO}_{2}$ Janus particles (Figures 2h-j).

A Janus distribution of $\mathrm{Pt}$, which catalyses the decomposition of $\mathrm{H}_{2} \mathrm{O}_{2}$, on the surface of the microparticles creates asymmetric gradients around the $\mathrm{Pt}-\mathrm{SiO}_{2}$ particles. These in turn develop asymmetric flow fields, which result in the self-phoresis of the particles (19). To evaluate our galvanic approach to microswimmer fabrication, we perform particle tracking experiments in fuel-rich environments. We track the trajectories of the bulk-Pt-functionalised and Janus-Ptfunctionalised microparticles and quantify their short-time mean squared displacements (MSD, Figure 4a). We then evaluate the particle velocities from their short-time MSDs using $\left\langle\Delta x^{2}\right\rangle=$ $4 D_{T} \Delta \tau+V^{2} \Delta \tau^{2}$. This analysis requires that rotational diffusion does not influence the motion of the microswimmers, i.e. that $\tau<<\tau_{R}$, where $\tau_{R}$ is the characteristic time of rotation (20) (see Text S4 for discussion). We therefore compute the MSD over 2s (20 data points at 10 FPS), noting that $\tau_{R}$ is approximately $50 \mathrm{~s}$.

To determine the efficacy of our synthetic protocols to obtain microswimmers, we evaluate the active Pèclet numbers $(\mathrm{Pe})$ of the unfunctionalised $\mathrm{SiO}_{2}$, bulk Pt-functionalised $\mathrm{SiO}_{2}$, and the Janus Pt-functionalised $\mathrm{SiO}_{2}(2 I) . P e \propto \frac{V}{\sqrt{D_{T} D_{R}}}$ determines the ratio of the ballistic ( $V$ is the propulsion speed) and diffusive (with $D_{T}$ and $D_{R}$ the translational and rotational diffusiv- 


\section{a}

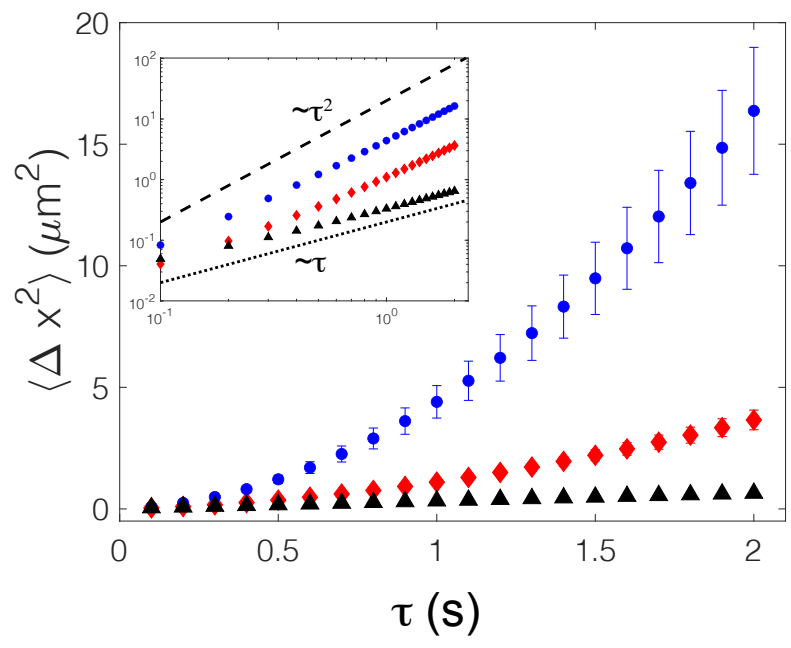

b

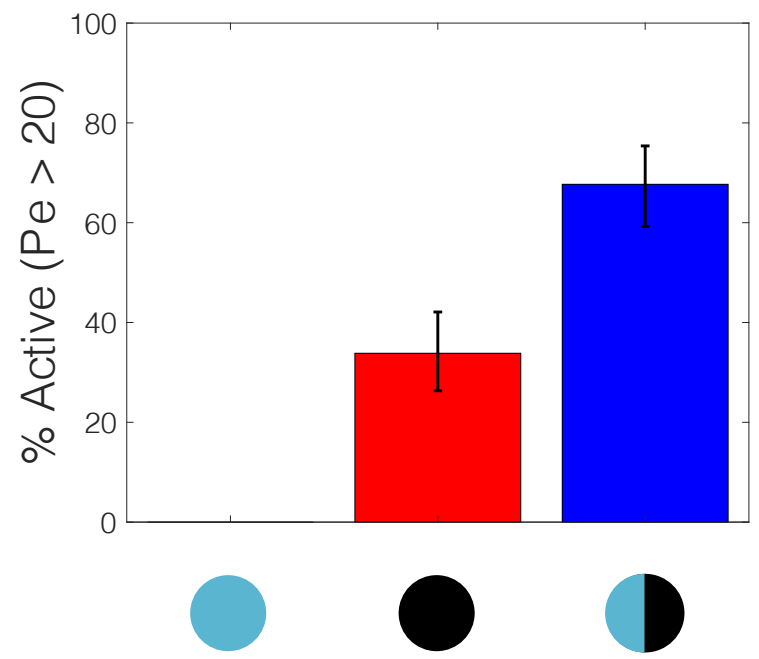

Figure 4: a. Short-time mean squared displacements (MSD). Unfunctionalised $\mathrm{SiO}_{2}$ (black triangles), bulk-functionalised $\mathrm{Pt}-\mathrm{SiO}_{2}$ (red diamonds), and Janus $\mathrm{Pt}_{-} \mathrm{SiO}_{2}$ ( $\mathrm{Pe}>20$, blue circles). The inset shows the MSD on a log-log scale. Error bars are the standard error $b$. Active particle yields (Pe $>20)$ from different synthetic routes (colours as in a.). The error bars correspond to 95\% confidence intervals, which were obtained with bootstrapping. All particle tracking investigations were performed using $0.5 \mathrm{v} / \mathrm{v} \% \mathrm{H}_{2} \mathrm{O}_{2}$ fuel concentration, and statistics were gathered from at least 130 particle trajectories.

ities, respectively) components of motion, and provides a measure of the relative importance of directed motion, ergo, the proportion of active particles. Setting a threshold $P e>20$, we find that $68 \%$ of the Janus Pt-functionalised particles are active, providing an indirect measure of the yield of our microswimmer fabrication protocol (Figure $4 \mathrm{~b}$ ). We note that even without the asymmetric functionalisation provided by the Pickering wax-emulsions, 34\% of the bulk Pt-functionalised particles surpass the $P e>20$ threshold. Spontaneous symmetry breaking of a concentration field and swimming behaviour has also been theorised for symmetric particles at $P e=4(22,23)$. However, in our case, the bulk-grown films possess inherent asymmetries, which we hypothesise are sufficient to induce minor gradients and thus particle motion. Nonetheless, the doubling in the proportion of microswimmers obtained by purposely 
introducing asymmetry as well as the higher overall fitted velocities (unfunctionalised $\mathrm{SiO}_{2}=$ $[0.077 ; 0.096] \mu \mathrm{ms}^{-1}$, bulk $\mathrm{Pt}=[0.63 ; 0.81] \mu \mathrm{ms}^{-1}$, Janus $\mathrm{Pt}=[1.2 ; 1.6] \mu \mathrm{ms}^{-1}-95 \%$ confidence intervals from bootstrapping) highlight the importance of the anisotropy obtained from using Pickering-wax emulsions.

In summary, we have demonstrated galvanic electrochemistry as a viable approach to fabricate Pt-based microswimmers in a scalable fashion by combination with the Pickering-wax emulsion technique. Interestingly, we find that the inherent asymmetry of the produced films also leads to motion of some particles functionalised by bulk stirring. Nonetheless, introducing asymmetry with Pickering wax emulsions doubles the proportion of microswimmers obtained growing Pt films and leads to a two-fold increase of the propulsion velocity. By using copper as a sacrificial template, the method minimises the use of expensive and toxic noble metal precursors, and the rapid complexation of copper by amine groups reduces synthesis time. Complexing first the metal ions before addition of a strong reducing agent also prevents the uncontrolled formation of nanoparticles in solution, providing a further handle on the synthesis of the metal films as well as minimising nanoparticle emissions (24). By exploiting galvanic electrochemistry, the method presented here can also be extended to a range of metals, suggesting a versatile approach to obtain large quantities of a range of functional Janus particles.

\section{Experimental Section}

Copper seeding onto $\mathrm{SiO}_{2}$ microparticles $\mathrm{SiO}_{2}$ microparticles were modified in water for reasons outlined in the Supporting Information (Text S3). Briefly, $125 \mathrm{mg}$ of $4.16 \mu \mathrm{m} \mathrm{SiO}{ }_{2}$ microparticles ( $\mathrm{SD}=0.19 \mu \mathrm{m}$, microparticles $\mathrm{GmbH}$ ) were dispersed in $49 \mathrm{~mL}$ double distilled water, and then $1 \mathrm{~mL}$ of $\mathrm{NH}_{4} \mathrm{OH}$ was added under stirring (1000 RPM). After 5 minutes, $90 \mu \mathrm{L}$ of APTES was added dropwise, and the suspension was left to stir for a further 1 hour. The APTES-modified particles were then washed 4 times by centrifugation (1300 RPM, 1 minute) 
with double-distilled water $(50 \mathrm{~mL})$. The suspension was then made up to $45 \mathrm{~mL}$ with doubledistilled water, and $5 \mathrm{~mL}$ of an aqueous $2 \mathrm{mg} / \mathrm{mL} \mathrm{CuSO}_{4}$ solution was added dropwise under stirring (1000 RPM) and left for 1 hour. The $\mathrm{Cu}(\mathrm{II})$-APTES $\mathrm{SiO}_{2}$ microparticles were then washed 4 times with double-distilled water $(50 \mathrm{~mL})$ by centrifugation (1300 RPM, 1 minute). The solution was then made up to $22.5 \mathrm{~mL}$ with double distilled water, and degassed for 30 minutes with $\mathrm{N}_{2}$ under stirring (1000 RPM). $2.5 \mathrm{~mL}$ of a $1 \mathrm{M}$ aqueous $\mathrm{NaBH}_{4}$ solution was then added dropwise, and the reaction proceeded for 30 minutes under continuous degassing. The resulting brown $\mathrm{Cu}^{0}-\mathrm{SiO}_{2}$ particles were then washed 3x in degassed, double-distilled water $(50 \mathrm{~mL})$, before making up the suspension to $150 \mathrm{~mL}$ with degassed, double-distlled water.

Platinum film growth from copper seeds $50 \mathrm{~mL}$ of an aqueous $0.33 \mathrm{mM}(34 \mathrm{mg}) \mathrm{H}_{2} \mathrm{PtCl}_{6} \cdot 6 \mathrm{H}_{2} \mathrm{O}$ solution was added to the stirred $150 \mathrm{~mL} \mathrm{Cu}-\mathrm{SiO}_{2}$ particle suspension (1000 RPM) under continuous degassing. The galvanic exchange proceeded for 30 minutes, before dropwise addition of $1.35 \mathrm{~mL}$ formaldehyde to the suspension to initiate the auto-catalytic growth of the Pt films. The vessel was then degassed vigorously for a further 5 minutes, then left to react overnight in a sealed vessel. The resulting $\mathrm{Pt}-\mathrm{SiO}_{2}$ microparticles were then washed $4 \mathrm{x}$ with double-distilled water.

Preparation of $\mathrm{SiO}_{2}$-wax colloidosomes $250 \mathrm{mg}$ of $\mathrm{SiO}_{2}$ particles were dispersed in didodecyldimethylammonium bromide (DDAB) solutions with concentrations corresponding to an approximate surfactant monolayer coverage on all particles $(5 \mathrm{~mL}, 0.0054 \mathrm{mg} / \mathrm{mL})(16)$. Wax (230 mg) was added to the suspension, and then placed in an silicone oil bath at $75{ }^{\circ} \mathrm{C}$, and first stirred at 3000 RPM for 15 minutes after the wax had melted with a T-25 digital ULTRATURRAX. After this initial dispersion step, the suspension was then homogenized under vigorous stirring for $160 \mathrm{~s}$ (11000 RPM) (17). After homogenization, the hot, wax-in-water $\mathrm{SiO}_{2}$ particle-stabilised Pickering emulsion was then rapidly cooled in an ice-bath to obtain solidified $\mathrm{SiO}_{2}$-wax colloidosomes. The colloidosomes were then washed consecutively by gravitational 
sedimentation with distilled water to remove excess wax and unattached particles ( 2 times, 50 $\mathrm{mL})$, a $0.1 \mathrm{M} \mathrm{NaCl}$ solution to remove the DDAB cationic surfactant $(2 \mathrm{x}, 50 \mathrm{~mL})$, then distilled water $(3 \mathrm{x}, 50 \mathrm{~mL})$ to remove any salt.

Asymmetric platinum film growth on $\mathrm{SiO}_{2}$ microparticles The washed $\mathrm{SiO}_{2}$-wax colloidosomes were dispersed in an aqueous $\mathrm{NH}_{4} \mathrm{OH}$ solution $(100 \mathrm{~mL}, 0.6 \mathrm{v} / \mathrm{v} \%)$ under gentle agitation using an orbital shaker. $360 \mu \mathrm{L}$ of APTES was then added dropwise to this suspension, and left for 1 hour under continued shaking (350 RPM). The APTES-modified $\mathrm{SiO}_{2}$-wax colloidosomes were then collected by vacuum filtration on ashless filter paper and washed with copious amounts of distilled water $(300 \mathrm{~mL})$. The collected colloidosomes were then left to dry in a vacuum oven at $40^{\circ} \mathrm{C}$, weighed, then dispersed in $50 \mathrm{~mL} \mathrm{CHCl}_{3}$ and left stirring for 1 hour to dissolve the wax. The Janus-modified APTES-SiO ${ }_{2}$ microparticles were then collected by vacuum filtration on a PTFE filter membrane (pore size $1.5 \mu \mathrm{m}$ ) by copious washing first by $\mathrm{CHCl}_{3}(150 \mathrm{~mL})$ then $\mathrm{EtOH}(50 \mathrm{~mL})$. The particles were again dried in a vacuum oven and weighed. The yield of Janus particles was typically on the order of 50\% (125 mg). Pt films were then asymmetrically grown on the Janus APTES-SiO ${ }_{2}$ microparticles as described above.

Particle tracking experiments Particle tracking experiments were performed on using a Nikon Upright LV150N microscope (20x). Stock $\mathrm{H}_{2} \mathrm{O}_{2}$ (30 v/v\%, Acros Organics) was added to dilute particle suspensions of the Pt-functionalised microswimmers to obtain $300 \mathrm{uL}$ of a fuel rich $\left(0.5 \mathrm{v} / \mathrm{v} \% \mathrm{H}_{2} \mathrm{O}_{2}\right)$ solution. $280 \mu \mathrm{L}$ thereof was then pipetted into a flow-through cell (cell 137-QS; Hellma Analytics) with a light path length of $1 \mathrm{~mm}$. Videos were taken at $10 \mathrm{fps}$ on a Hamamatsu C14440-20UP digital camera. Particle trajectories were then analysed using the MATLAB implementation of the Crocker and Grier IDL particle tracking method (25) and in-house MATLAB scripts. 


\section{Acknowledgements}

The authors thank David Häberlin and Aaron Iten for their exploratory investigations at the beginning of the project. They also thank Dr. M. Hu for his assistance with the FIB-SEM imaging.

\section{Author Contribution Statement}

Author contributions are defined based on the CRediT (Contributor Roles Taxonomy). Conceptualization: M.R.B., L.I. Formal Analysis: M.R.B., F.G. Funding acquisition: F.G., L.I. Investigation: M.R.B., N.R., A.F Methodology: M.R.B., N.R. Software: M.R.B. Supervision: M.R.B., F.G., L.I. Validation: M.R.B., N.R., A.F. Visualization: M.R.B., F.G., L.I. Writing original draft: M.R.B., L.I. Writing - review and editing: M.R.B., N.R., F.G., L.I. 


\section{References}

1. S. Ramaswamy, Annual Review of Condensed Matter Physics 2010, 1323.

2. M. E. Cates, J. Tailleur, Annual Review of Condensed Matter Physics 2015, 6, 1219.

3. P. Díez, E. Lucena-Sánchez, A. Escudero, A. Llopis-Lorente, R. Villalonga, R. MartínezMáñez, ACS Nano 2021, 15, 34467.

4. L. Wang, A. Kaeppler, D. Fischer, J. Simmchen, ACS Applied Materials and Interfaces 2019, 11, 3632937.

5. V. V. Singh, F. Soto, K. Kaufmann, J. Wang, Angewandte Chemie-International Edition 2015, 54, 236896.

6. Y. Alapan, B. Yigit, O. Beker, A. F. Demirörs, M. Sitti, Nature Materials 2019, 18, 11 1244.

7. K. Yuan, M. Pacheco, B. Jurado-Sánchez, A. Escarpa, Advanced Intelligent Systems 2021, 2100002.

8. M. N. Popescu, Langmuir 2020, 36, 256861.

9. J. Zhang, F. Mou, Z. Wu, S. Tang, H. Xie, M. You, X. Liang, L. Xu, J. Guan, ACS Applied Materials and Interfaces 2019, 11, 1816639.

10. A. B. Pawar, I. Kretzschmar, AIChE Annual Meeting, Conference Proceedings 2008, , 22 355.

11. R. J. Archer, A. J. Parnell, A. I. Campbell, J. R. Howse, S. J. Ebbens, Advanced Science 2018, 5, 21 . 
12. G. Bystrzejewska-Piotrowska, J. Golimowski, P. L. Urban, Waste Management 2009, 29, 9 2587.

13. J. Bastos-Arrieta, C. Bauer, A. Eychmüller, J. Simmchen, Journal of Chemical Physics 2019, $150,14$.

14. B. Modec, N. Podjed, N. Lah, Molecules 2020, 25, 7.

15. A. Perro, F. Meunier, V. Schmitt, S. Ravaine, Colloids and Surfaces A: Physicochemical and Engineering Aspects 2009, 332, 157.

16. S. Kord Daroun Kalai, Synthesis of silica Janus nanoparticles with metal semishells, 2019, URL https://refubium.fu-berlin.de/handle/fub188/24535.

17. K. Lebdioua, A. Aimable, M. Cerbelaud, A. Videcoq, C. Peyratout, Journal of Colloid and Interface Science 2018, 520127.

18. M. R. Bailey, F. Grillo, N. D. Spencer, L. Isa, arXiv 2021, 1-36.

19. J. Anderson, Annual Review of Fluid Mechanics 1989, 21, 161.

20. S. Ketzetzi, J. De Graaf, R. P. Doherty, D. J. Kraft, Physical Review Letters 2020, 124, 4 48002.

21. C. Bechinger, R. Di Leonardo, H. Löwen, C. Reichhardt, G. Volpe, G. Volpe, Reviews of Modern Physics 2016, 88, 4.

22. R. Golestanian, Physical Review Letters 2009, 102, 18188305.

23. S. Michelin, E. Lauga, D. Bartolo, Physics of Fluids 2013, 25, 6.

24. M. Bundschuh, J. Filser, S. Lüderwald, M. S. McKee, G. Metreveli, G. E. Schaumann, R. Schulz, S. Wagner, Environmental Sciences Europe 2018, 30, 1. 
25. J. C. Crocker, D. G. Grier, Journal of Colloid and Interface Science 1996, 179, 1298. 